\title{
Controlling vortex chirality and polarity by geometry in magnetic nanodots
}

Sebastia Agramunt-Puig, Nuria Del-Valle, Carles Navau, and Alvaro Sanchez

Citation: Appl. Phys. Lett. 104, 012407 (2014);

View online: https://doi.org/10.1063/1.4861423

View Table of Contents: http://aip.scitation.org/toc/apl/104/1

Published by the American Institute of Physics

\section{Articles you may be interested in}

The design and verification of MuMax3

AIP Advances 4, 107133 (2014); 10.1063/1.4899186

Current controlled random-access memory based on magnetic vortex handedness

Applied Physics Letters 93, 142508 (2008); 10.1063/1.2998584

Controllable switching of vortex chirality in magnetic nanodisks by a field pulse

Applied Physics Letters 92, 012503 (2008); 10.1063/1.2829795

Eigenfrequencies of vortex state excitations in magnetic submicron-size disks

Journal of Applied Physics 91, 8037 (2002); 10.1063/1.1450816

Magnetic switching of single vortex permalloy elements

Applied Physics Letters 79, 3113 (2001); 10.1063/1.1410873

Electric field control of Skyrmions in magnetic nanodisks

Applied Physics Letters 108, 152403 (2016); 10.1063/1.4945738

\section{Scilight}

Sharp, quick summaries illuminating the latest physics research

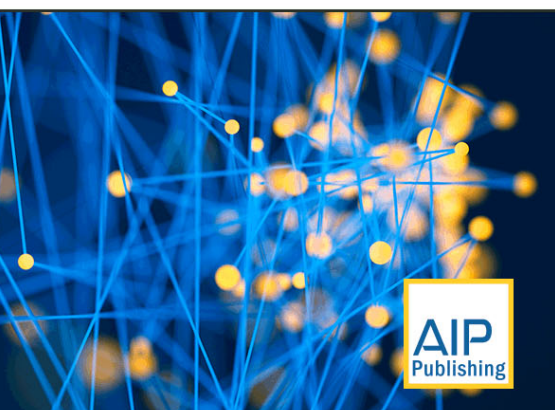




\title{
Controlling vortex chirality and polarity by geometry in magnetic nanodots
}

\author{
Sebastia Agramunt-Puig, Nuria Del-Valle, Carles Navau, and Alvaro Sanchez \\ Departament de Física, Grup d'Electromagnetisme, Universitat Autònoma de Barcelona, 08193 Bellaterra, \\ Barcelona, Catalonia, Spain
}

(Received 19 September 2013; accepted 20 December 2013; published online 9 January 2014)

\begin{abstract}
The independent control of both vortex chirality and polarity is a significant challenge in magnetic devices based on nano-sized magnetic vortex structures. By micromagnetic simulations here, we show that in soft ferromagnetic nanodots with an adequate modulated thickness, the desired combination of chirality and polarity can be achieved just by changing the direction of the in-plane applied magnetic field. Despite the complex behavior, the vortex chirality and polarity control can be summarized in two simple rules that can thus become a useful tool for designing magnetic devices. (C) 2014 AIP Publishing LLC. [http://dx.doi.org/10.1063/1.4861423]
\end{abstract}

In thin soft ferromagnetic disks (dots) of submicrometer size, the vortex-state magnetization is the spin configuration with the lowest energy at zero applied magnetic field. ${ }^{1}$ This vortex state is characterized by an in-plane curling magnetization (with a given chirality [C] either clockwise [CW] or counterclockwise $[\mathrm{CCW}]$ ) and a nanometer size central region with an out-of-plane magnetization named vortex core (with an up or down polarity $[\mathrm{P}])^{2}{ }^{2}$ This magnetization pattern represents a compromise between the different competing contributions of the total energy. In the case of very soft (negligible magnetic anisotropy) materials without external field, these contributions are the magnetostatic (or stray field) and exchange energies. The curling in-plane magnetization closing the magnetic flux reduces the magnetostatic energy, but in the vortex core the magnetization turns out of plane because the short-range exchange interaction favors a parallel alignment between neighboring spins.

The multiple stable ground states of vortices have led to the study of these magnetization patterns as potential multibit memory cells. ${ }^{3}$ This application requires independent control of both vortex chirality and polarity. ${ }^{4}$ In the literature, there is a large number of works focused on the control and/or modification of the vortex state in soft magnetic dots. On the one hand, there is much interest to achieve the vortex core polarity switching. This reversal process can be due to non-resonant single field/current pulse ${ }^{5}$ or by means of applied magnetic bursts ${ }^{6}$ or spin-polarized currents ${ }^{7}$ inducing an oscillatory mode in the vortex core around its equilibrium position (gyrotropic mode ${ }^{8}$ ), or else to spin waves ${ }^{9}$ inducing some excitation in the azimuthal modes. ${ }^{10}$ On the other hand, some works show how to control the chirality of the vortex by introducing some asymmetries in the shape of dot, ${ }^{11-14}$ in its magnetic properties, ${ }^{15}$ or in the applied field distribution. ${ }^{16-18}$ Some of them also control the vortex polarity by adding an out-of-plane magnetic field ${ }^{13}$ or by using non practical complex geometry. ${ }^{14} \mathrm{~A}$ remaining goal is to achieve the simultaneous control of chirality and polarity in dots with practical and existing geometries by adjusting a single parameter. Very recently, some authors ${ }^{4,19}$ have presented a way to control the chirality of the vortex by modulating the thickness of the dots. Here, we present a strategy to control polarity as well. The desired combination of chirality and polarity at will is achieved in a practical geometry by changing only a simple parameter, the direction of the inplane applied magnetic field.

In our own-coded micromagnetic simulations, the soft ferromagnetic material is discretized into a uniform array of cubic cells (micromagnetic cells) in which the effective field $\mathbf{H}_{\text {eff }}$ (the sum of applied, stray, and exchange fields) $)^{20,21}$ is calculated by a finite-difference method. ${ }^{21-23}$ The stray field is obtained directly using volume (micromagnetic cell) averaged expressions. ${ }^{24}$ Calculation time is reduced by computing the stray field from farther cells as the field due to a larger effective cell whose magnetization is the average of the small contained ones. ${ }^{25}$ The exchange field is obtained using a six-neighbor scheme with appropriate boundary conditions. ${ }^{26}$ Brown's static equations ${ }^{20}$ are solved using an iterative algorithm. Let $\mathbf{m}_{i}\left(\mathbf{r}_{k}\right)$ be the unitary vector pointing the direction of the magnetization at each micromagnetic cell $k$ located at position $\mathbf{r}_{k}$ for the iteration step $i$. Then, the effective field $\mathbf{H}_{\text {eff }, i}\left(\mathbf{r}_{k}\right)$ is computed and the new $\mathbf{m}_{i+1}\left(\mathbf{r}_{k}\right)$ is set to point in the same direction as the local effective field calculated. This process is repeated until the maximum change of angle (in spherical coordinates) with respect to the previous iteration step is less than some tolerance $\left(10^{-4} \mathrm{rad}\right)$. When the applied field value is changed, a small random angle with uniform distribution and maximum amplitude $10^{-4} \mathrm{rad}$ is applied to the magnetization of all cells. This allows to randomly break the symmetries that could lead to metastable situations.

We consider a Permalloy cylinder with isotropic exchange constant $A=1.3 \times 10^{-11} \mathrm{~J} / \mathrm{m}$, saturation magnetization $M_{\mathrm{s}}=8 \times 10^{5} \mathrm{~A} / \mathrm{m}$, and, for simplicity, a negligible crystalline anisotropy. The sample is discretized in $6 \mathrm{~nm}$ sided cubic cells, according to the exchange length ${ }^{22}$ $\left(l_{\mathrm{ex}}=\sqrt{2 A / \mu_{0} M_{\mathrm{s}}} \approx 5.7 \mathrm{~nm}\right)$, to obtain sufficiently accurate results. ${ }^{27}$ The simulated dot (Fig. 1(a)) has a diameter $a=360 \mathrm{~nm}$, a thin half part with a thickness $b=18 \mathrm{~nm}$ at $x<0$, and a thicker half of thickness $b+c=24 \mathrm{~nm}$ at $x>0$ separated by a middle edge. The in-plane ( $x y$-plane) applied magnetic field forms an angle $\theta$ with the $x>0$ axis. The dot is initially saturated in the applied field direction and then the field is decreased. Around zero applied field, a vortex state appears with some polarity and chirality.

Figure 1(c) shows a summary of the different vortex states obtained from the numerical results as a function of $\theta$. To understand these general results, we analyze the particular 


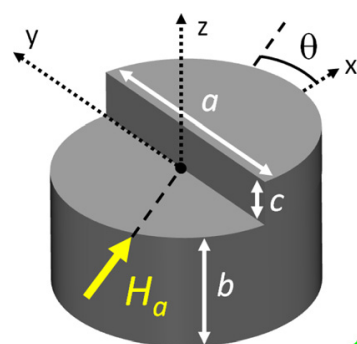

(a)
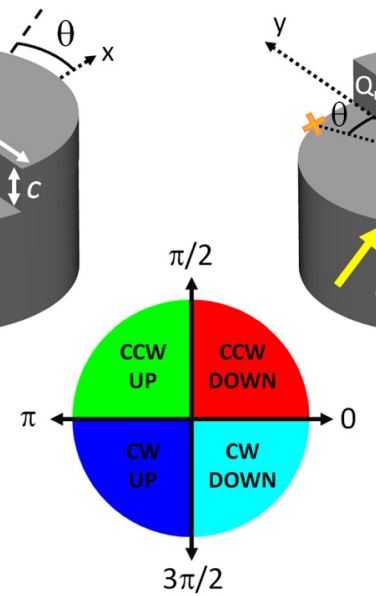

(c)
FIG. 1. (a) Sketch of a soft ferromagnetic dot with two half parts of different thicknesses. The dot is subjected to an in-plane applied magnetic field that forms an angle $\theta$ with the $x>0$ axis. (b) The same dot as (a) in the case that the magnetization presents a $\mathrm{C}$-state configuration in the thicker region, for which there appear two effective point magnetic charges with opposite sign $Q_{\mathrm{m} 1}$ and $Q_{\mathrm{m} 2}$ in the edge surface. Yellow $\mathrm{X}$ marks the vortex nucleation point (perpendicular to the applied field direction). (c) Vortex chiralities and polarities obtained from numerical results as a function of the angle $\theta$ between the applied magnetic field and the $x>0$ axis.

cases of $\theta=\pi / 4$ (Fig. 2) and $3 \pi / 4$ (Fig. 3), in which the four different vortex states of Fig. 1(c) appear. Figure 2(a) shows the hysteresis loop of the magnetization component parallel to the initial saturation field, $M_{\|}$, for the decreasing applied-field process with $\theta=\pi / 4$. When the applied field is decreased after saturation, the magnetic moments of the thicker zone are the first ones that change their orientation to reduce the magnetostatic energy. These moments trigger a $\mathrm{C}$-state (a preceding state of the vortex configuration), following the rounded border of the sample but keeping mainly the direction of the applied field (panel (1) of Fig. 2(b)). Because of the modulated thickness, when the C-state is formed there appear some magnetic pole densities (positive and negative) in the flat surface of the middle edge (see the right part of panel (1) in Fig. 2(b)). These poles will create a net out-of-plane magnetic field, in particular, in the region where the vortex will nucleate (at the outer edge of the thinner region perpendicular to the applied field direction), thus determining the polarity of the vortex core. The direction of the C-state determines the chirality of the subsequent vortex state and also the sign of the induced magnetic poles and thus the vortex polarity. For $\theta=\pi / 4$ (and for $0<\theta<\pi / 2$ ), the vortex state is created with CCW chirality and down (core pointing $-z$ ) polarity (panel (2) of Fig. 2(b)). The field created by the magnetic poles at the nucleation point is negative because of the proximity of the positive poles (panel (1) of Fig. 2(b)).

The process of increasing applied field (started with negative saturation) of Fig. 2 is equivalent to the initially positive saturated $\theta=5 \pi / 4$. In this case (also for $\pi<\theta<3 \pi / 2$ ), the vortex appears with $\mathrm{CW}$ chirality and up (core pointing $+z$ ) polarity, the opposite situation to the case $\theta=\pi / 4$ (panels (1) and (3) of Fig. 2(b)). Now, since the chirality is inverted, the sign of the magnetic poles also changes and the polarity flips. Although the nucleation points are the same in the (a)

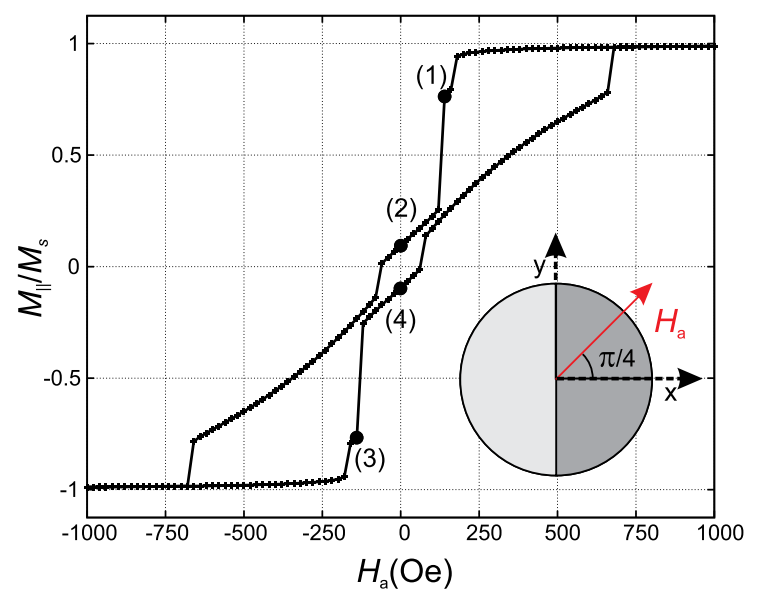

(b)
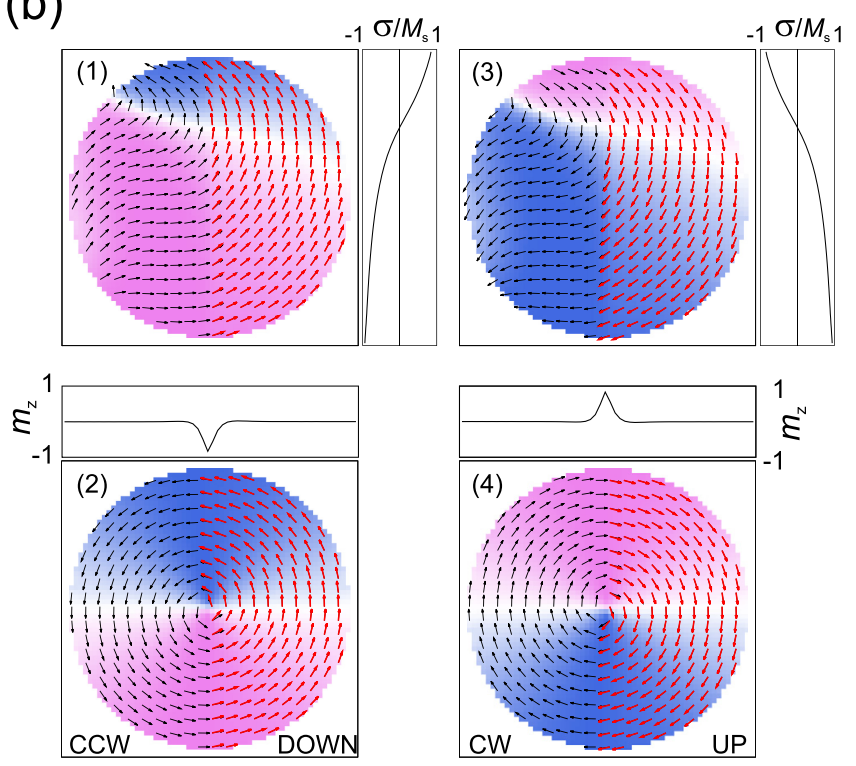

FIG. 2. (a) Calculated magnetization hysteresis loop of a Permalloy dot of Fig. 1(a) with diameter of $360 \mathrm{~nm}$ and thicknesses of $18 \mathrm{~nm}$ and $24 \mathrm{~nm}$. The inset shows a scheme of the dot and the direction of the initial saturation field ( $\pi / 4$ with respect to the positive $x$ axis). The thicker part of the dot $(x>0)$ is dark gray colored. (b) Magnetization distribution of the marked points in (a). Black arrows represent the in-plane magnetization and violet and blue colors indicate $m_{x}>0$ and $m_{x}<0$, respectively. Red arrows indicate the in-plane magnetization of the thicker part. The pole density distribution $\sigma$ along the middle edge is plotted in the right part of the upper graphs. In the upper part of the lower graphs, the out-of-plane magnetization $m_{z}$ along the line $y=0$ and $z=-3 \mathrm{~nm}$ is also plotted. The number of plotted spins is reduced for clarity.

decreasing and increasing field branches, since the chiralities are $\mathrm{CCW}$ and $\mathrm{CW}$, respectively, the sign of the induced magnetic poles is inverted and so is the polarity of the core (panels (1) and (3)). This means that in a magnetization reversal loop that reaches saturation both chirality and polarity are opposite in the two branches of the loop but the handedness, defined as the product of chirality times polarity (where $\mathrm{CW} \rightarrow 1, \mathrm{CCW} \rightarrow-1$, up $\rightarrow 1$, and down $\rightarrow-1$ ), does not change. Therefore, for a given applied field with a determined vortex polarity, one way to switch it is to reverse the applied field (after reversed saturation).

In Fig. 3, we show the results of decreasing and increasing applied-field processes for $\theta=3 \pi / 4$ (and for $\pi / 2<\theta<\pi$ ) and $\theta=7 \pi / 4$ (also for $3 \pi / 2<\theta<2 \pi$ ), 
(a)

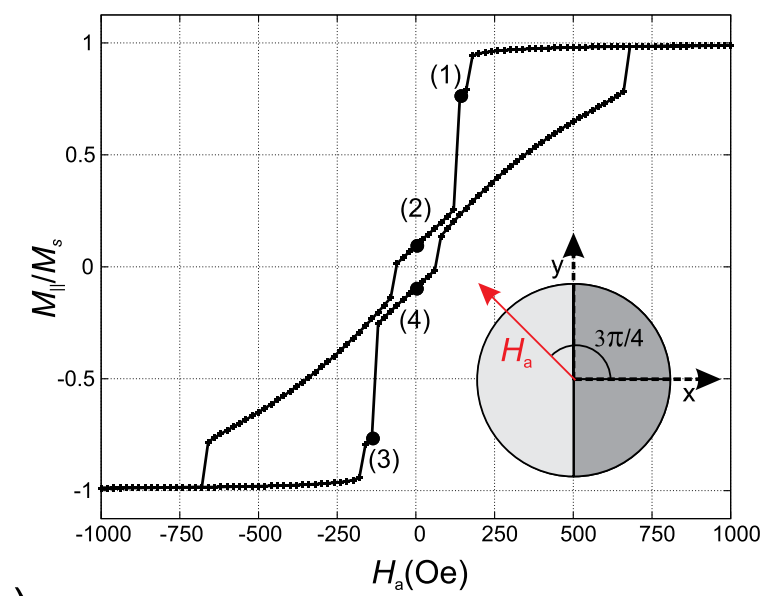

(b)
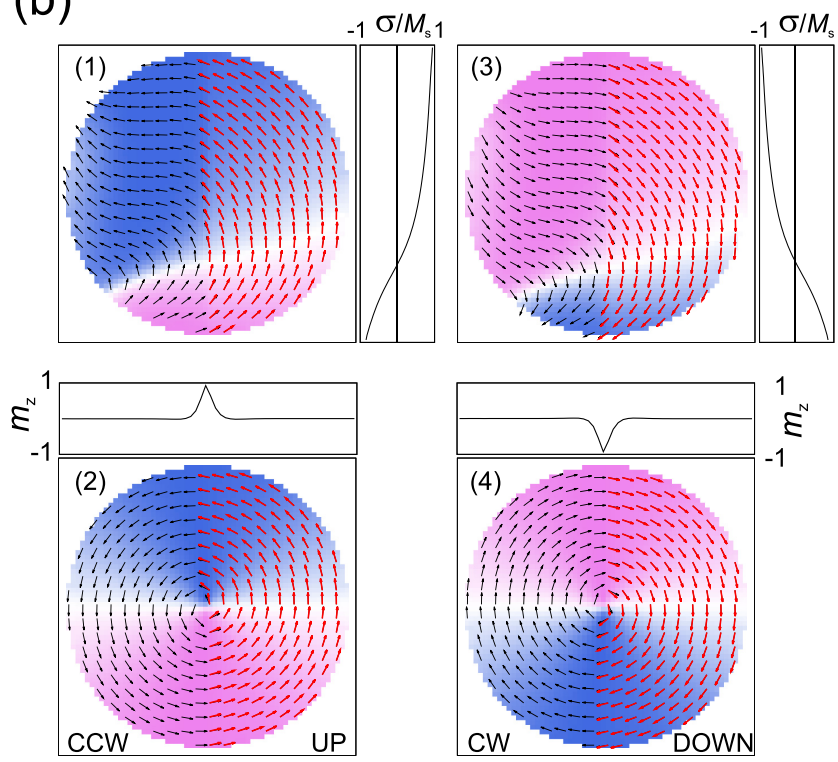

FIG. 3. The same as Fig. 2 but with $\theta=3 \pi / 4$.

respectively. By symmetry, the hysteresis loop (Fig. 3(a)) and the chiralities of the vortex states (Fig. 3(b)) are the same as in Fig. 2. However, the vortex nucleation point has changed and the nearest magnetic poles too (panels (1) and (3) of Fig. 3(b)), so the polarities are opposite to the ones of Fig. 2.

In the particular cases of $\theta=\pi / 2$ and $3 \pi / 2$, the obtained chirality in the vortex state is always CCW and $\mathrm{CW}$, respectively, but in both cases the polarity is undetermined because the effects of the magnetic poles are compensated by symmetry. For $\theta=0$ and $\pi$, the vortex state configuration can be any of the different four configurations because both polarity and chirality are undetermined.

The middle edge has not necessarily to be in the center of the dot to determine both chirality and polarity. We obtain similar results to Fig. 1(c) (not shown) when the thicker part begins at $x=-a / 4$ (three quarter parts of the dot) or at $x=a / 4$ (one quarter), for example, showing the robustness of the vortex control.

The control of chirality and polarity in this kind of dots with modulated thickness can be summarized into two simple rules:
(1) The chirality of the curling magnetization is controlled by both the position of the thicker part and the sign of the applied field component parallel $\left(H_{a \|}\right)$ to the middle edge. If the thicker zone is placed at $x>0(x<0)$, for $H_{a \|}>0$ the chirality is CCW (CW) while for $H_{a \|}<0$ the chirality is $\mathrm{CW}(\mathrm{CCW})$.

(2) The vortex core polarity is controlled by the sign of nearest side poles (determined by the chirality) of the middle edge. If the sign is positive (negative), the vortex core is down (up).

A simple analytical model can capture the physics of the vortex-state determination in these dots. We consider the dot shown in Fig. 1(b) with the same dimensions as in the numerical case and with the in-plane applied field $\mathbf{H}_{a}=H_{a}(\cos \theta \hat{x}+\sin \theta \hat{y})$. As first approximations, we consider that the vortex nucleates at $x=x_{N}=-\frac{a}{2}|\sin \theta|$, $y=y_{N}=\operatorname{Sign}[\sin \theta] \frac{a}{2} \cos \theta$, and $z=z_{N} \in[0,-b]$ (at the thinner edge perpendicular to the field direction), and the magnetic pole densities induced in the $\mathrm{C}$-state are always assumed as two point-like opposite magnetic charges $Q_{\mathrm{m} 1}$ and $Q_{\mathrm{m} 2}$ (Fig. 1(b)). The sign of the magnetic charges is obtained by the sign of the applied-field component parallel to the flat surface of the middle edge $Q_{\mathrm{m} 1}=\operatorname{Sign}[\sin \theta] M_{\mathrm{s}} c \frac{a}{2}$ and $Q_{m 2}=-Q_{\mathrm{m} 1}$, so the chirality is $C=\operatorname{Sign}[\sin \theta]$. The sign of out-of-plane component of the magnetic field created by these point charges (analytically obtained by Biot-Savart equations) at the nucleation points (a function of $\theta$ ) determines the polarity of the vortex $P=\operatorname{Sign}\left[H_{z}\left(x_{N}(\theta), y_{N}(\theta), z_{N}(\theta)\right)\right]$.

Figure 4(a) shows the evolution in $\theta$ of the out-of-plane field by the two charges at the top $\left(z_{N}=0\right.$, solid $)$ and bottom

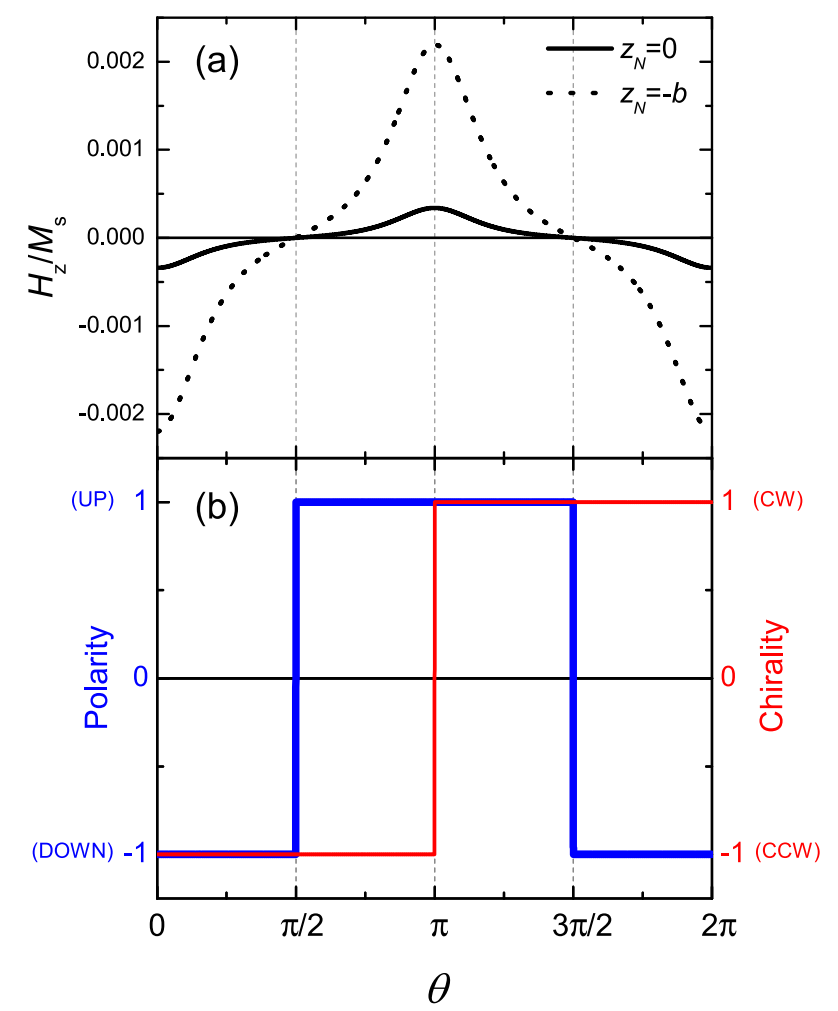

FIG. 4. (a) Out-of-plane magnetic field created by the two opposite magnetic charges at the nucleation point $\left(x_{N}, y_{N}\right)$ with $z_{N}=0$ (solid) and $z_{N}=-b$ (dot) as a function of $\theta$. (b) Polarity (thick blue) and chirality (thin red) of the vortex state as a function of $\theta$. 
$\left(z_{N}=-\mathrm{b}\right.$, dot $)$ in the thin region. In both cases and in the intermediate ones, the trend is the same. For $0<\theta<\pi / 2$ and $3 \pi / 2<\theta<2 \pi$ the $\mathrm{z}$-field is negative because the positive charge $\left(Q_{\mathrm{m} 1}\right.$ and $Q_{\mathrm{m} 2}$, respectively) is the nearest one. When $\pi / 2<\theta<\pi$ and $\pi<\theta<3 \pi / 2$ the proximity of the negative charge $\left(Q_{\mathrm{m} 2}\right.$ and $Q_{\mathrm{m} 1}$, respectively) makes the field to be positive. At $\theta=\pi / 2$ and $3 \pi / 2$, the z-field is zero because the two opposite charges are equidistant from the nucleation point and their effects are compensated. Figure 4(b) presents a summary of the vortex chirality and polarity behaviors as a function of $\theta$. These results agree with the numerical ones obtained in Fig. 1(c).

In conclusion, both polarity and chirality of vortex state in magnetic dots with modulated thickness can be independently controlled by simply adjusting the in-plane applied field direction. Two simple rules allow to control the desired vortex state which can be useful in the design of magnetic devices.

We thank Spanish Projects NANOSELECT (CSD200700041) and MAT2012-35370 for financial support.

${ }^{1}$ R. P. Cowburn, D. K. Koltsov, A. O. Adeyeye, and M. E. Welland, Phys. Rev. Lett. 83, 1042 (1999).

${ }^{2}$ T. Shinjo, T. Okuno, R. Hassdorf, K. Shigeto, and T. Ono, Science 289, 930-932 (2000).

${ }^{3}$ S. Bohlens, B. Krüger, A. Drews, M. Bolte, G. Meier, and D. Pfannkuche, Appl. Phys. Lett. 93, 142508 (2008); K. Nakano, D. Chiba, N. Ohshima, S. Kasai, T. Sato, Y. Nakatani, K. Sekiguchi, K. Kobayashi, and T. Ono, ibid. 99, 262505 (2011); M. Goto, H. Hata, A. Yamaguchi, Y. Nakatani, T. Yamaoka, Y. Nozaki, and H. Miyajima, Phys. Rev. B 84, 064406 (2011).

${ }^{4}$ V. Uhlir, M. Urbanek, L. Hladik, J. Spousta, M.-Y. Im, P. Fischer, N. Eibagi, J. J. Kan, E. E. Fullerton, and T. Sikola, Nat. Nanotechnol. 8, 341 (2013).

${ }^{5}$ R. Hertel, S. Gliga, M. Fähnle, and C. M. Schneider, Phys. Rev. Lett. 98, 117201 (2007); Y. Liu, S. Gliga, R. Hertel, and C. M. Schneider, Appl. Phys. Lett. 91, 112501 (2007); K. Yamada, S. Kasai, Y. Nakatani, K. Kobayashi, and T. Ono, ibid. 93, 152502 (2008).

${ }^{6}$ B. Van Waeyenberge, A. Puzic, H. Stoll, K. W. Chou, T. Tyliszczak, R. Hertel, M. Fähnle, H. Brückl, K. Rott, G. Reiss, I. Neudecker, D. Weiss, C. H. Back, and G. Schütz, Nature 444, 461-464 (2006).
${ }^{7}$ K. Yamada, S. Kasai, Y. Nakatani, K. Kobayashi, H. Kohno, A. Thiaville, and T. Ono, Nature Mater. 6, 270-273 (2007).

${ }^{8}$ K. Y. Guslienko, K.-S. Lee, and S.-K. Kim, Phys. Rev. Lett. 100, 027203 (2008).

${ }^{9}$ M. Kammerer, M. Weigand, M. Curcic, M. Noske, M. Sproll, A. Vansteenkiste, B. Van Waeyenberge, H. Stoll, G. Woltersdorf, C. H. Back, and G. Schuetz, Nat. Commun. 2, 279 (2011).

${ }^{10}$ K. Y. Guslienko, A. N. Slavin, V. Tiberkevich, and S.-K. Kim, Phys. Rev. Lett. 101, 247203 (2008).

${ }^{11}$ S. Yakata, M. Miyata, S. Nonoguchi, H. Wada, and T. Kimura, Appl. Phys. Lett. 97, 222503 (2010).

${ }^{12}$ N. M. Vargas, S. Allende, B. Leighton, J. Escrig, J. Mejia-Lopez, D. Altbir, and Ivan K. Schuller, J. Appl. Phys. 109, 073907 (2011).

${ }^{13}$ M. Jaafar, R. Yanes, D. Perez de Lara, O. Chubykalo-Fesenko, A. Asenjo, E. M. Gonzalez, J. V. Anguita, M. Vazquez, and J. L. Vicent, Phys. Rev. B 81, 054439 (2010).

${ }^{14}$ V. Cambel and G. Karapetrov, Phys. Rev. B 84, 014424 (2011).

${ }^{15}$ Z. Zhong, H. Zhang, X. Tang, Y. Jing, L. Jia, and S. Liu, J. Magn. Magn. Mater 321, 2345-2349 (2009).

${ }^{16}$ M. Konoto, T. Yamada, K. Koike, H. Akoh, T. Arima, and Y. Tokura, J. Appl. Phys. 103, 023904 (2008).

${ }^{17}$ Y. Gaididei, D. D. Sheka, and F. G. Mertens, Appl. Phys. Lett. 92, 012503 (2008).

${ }^{18}$ S. Yakata, M. Miyata, S. Honda, H. Itoh, H. Wada, and T. Kimura, Appl. Phys. Lett. 99, 242507 (2011).

${ }^{19}$ G. Shimon, A. O. Adeyeye, and C. A. Ross, Phys. Rev. B 87, 214422 (2013).

${ }^{20}$ W. F. Brown, Jr., Magnetostatic Principles in Ferromagnetism (NorthHolland, Amsterdam, 1962).

${ }^{21}$ J. E. Miltat and M. J. Donahue, Numerical Micromagnetics: Finite Difference Methods (NIST, 2007); H. Kronmüller and S. S. P. Parkin, Handbook of Magnetism and Advanced Magnetic Materials (Wiley, 2007).

${ }^{22}$ H. Kronmüller and M. Fähnle, Micromagnetism and the Microstructure of Ferromagnetic Solids (Cambridge University Press, Cambridge, 2003).

${ }^{23}$ A. Aharoni, Introduction to the Theory of Ferromagnetism (Oxford University Press, Oxford, 1996).

${ }^{24}$ H. Fukushima, Y. Nakatani, and N. Hayashi, IEEE Trans. Magn. 34, 193 (1998).

${ }^{25}$ Our sample is also discretized into a second coarser uniform array of large cubic cells, where each coarse cell has $3 \times 3 \times 3$ small micromagnetic ones. The magnetostatic interaction between two micromagnetic cells is obtained from the averaged magnetization approximation of their coarse cells if they are, at least, neighbors of second order.

${ }^{26}$ M. J. Donahue and D. G. Porter, Physica B 343, 177 (2004).

${ }^{27}$ W. Rave, K. Ramstock, and A. Hubert, J. Magn. Magn. Mater. 183, 329 (1998); A. Aharoni, Physica B 306, 1 (2001). 